\title{
Contractile reserve assessed by dobutamine test identifies super-responders to cardiac resynchronization therapy
}

Dejan Vukajlovic ${ }^{1}$, Goran Milasinovic ${ }^{2}$, Lazar Angelkov${ }^{1}$, Velibor Ristic ${ }^{1}$, Milosav Tomovic ${ }^{1}$, Ruzica Jurcevic ${ }^{1}$, Petar Otasevic ${ }^{1}$

${ }^{1}$ Institute for Cardiovascular Diseases Dedinje, Belgrade, Serbia

2Pacemaker Center, Clinical Center of Serbia, Belgrade, Serbia

Submitted: 28 December 2012

Accepted: 1 April 2013

Arch Med Sci 2014; 10, 4: 684-691

DOI: 10.5114 /aoms.2014.40790

Copyright (c) 2014 Termedia \& Banach

\section{Abstract}

Introduction: In this study, we sought to determine whether myocardial contractile reserve (CR) assessed by dobutamine stress echocardiography (DSE) can identify patients who experience nearly complete normalization of left ventricular (LV) function after the implantation of a cardiac resynchronization therapy (CRT) pacemaker.

Material and methods: The study group consisted of 55 consecutive patients with non-ischemic dilated cardiomyopathy, LV ejection fraction (LVEF) $<35 \%$, and prolonged QRS complex duration, who were scheduled for CRT pacemaker implantation. The DSE $(20 \mu \mathrm{g} / \mathrm{kg} / \mathrm{min})$ was performed in all patients. The CR assessment was based on a change in the wall motion score index $(\triangle \mathrm{WMSI})$ and $\triangle \mathrm{LVEF}$ during DSE. Super-response was defined as an increase in LVEF to $>50 \%$ and reduction in left ventricular end-systolic dimension to $<40 \mathrm{~mm} 12$ months following the CRT implantation.

Results: A total of 7 patients (12.7\%) were identified as super-responders to CRT. When compared to non-super-responders, these patients had significantly higher values of the dobutamine-induced change in $\triangle$ WMSI $(1.031$ \pm 0.120 vs. $0.49 \pm 0.371, p<0.01)$, and $\Delta \mathrm{EF}(17.9 \pm 2.2$ vs. $8.8 \pm 6.2, p<0.01)$. Receiver operating characteristic analysis showed that dobutamine-induced changes in $\triangle \mathrm{WMSI} \geq 0.7$ and $\geq 14 \%$ for $\triangle \mathrm{EF}$ are the best discriminators for a super-response. Patients with $\Delta \mathrm{WMSI} \geq 0.7$ and $\Delta \mathrm{EF} \geq 14 \%$ are significantly less often hospitalized $(p<0.01)$ for worsening of heart failure during $28.5 \pm 3.0$ months of the follow-up.

Conclusions: Contractile reserve assessed by DSE can identify patients with dilated cardiomyopathy who are likely to experience near normalization of LV function following CRT.

Key words: cardiac resynchronization therapy, super-response, dobutamine stress echocardiography, contractile reserve.

\section{Introduction}

Although cardiac resynchronization therapy (CRT) is an established treatment option for patients with heart failure, depressed left ventricular ejection fraction (LVEF) and left bundle branch block (LBBB) or prolonged duration of the QRS complex [1, 2], a significant proportion of treated patients still lack functional, clinical and structural improvement. Identification of non-responders to CRT has been a matter of intensive
Corresponding author: Dejan Vukajlovic MD Institute for Cardiovascular Diseases Dedinje Milana Tepica 1

11000 Belgrade, Serbia Phone: +38111 3601682

E-mail: d.vukajlo@gmail.com 
investigation over the last several years. The absence of myocardial contractile reserve (CR), as assessed by dobutamine stress echocardiography (DSE), could detect patients who are less likely to respond beneficially to CRT [3].

On the other hand, some patients experience a complete functional and echocardiographic recovery during CRT [4]. These patients are termed super-responders. Female gender, non-ischemic etiology of heart failure, typical left bundle branch block morphology [5], as well as lower left ventricular dimensions and greater global longitudinal strain [6] were found to predict the super-response to CRT. However, to the best of our knowledge, there are no studies which evaluate a predictor role of $C R$ in these patients. In this study we sought to determine whether myocardial CR assessed by dobutamine stress echocardiography (DSE) can identify patients with dilated cardiomyopathy who experience nearly complete normalization of left ventricular (LV) function after the implantation of a CRT pacemaker.

\section{Material and methods}

The study group consisted of 55 consecutive patients with non-ischemic dilated cardiomyopathy, referred for CRT pacemaker implantation. The patients were included if they had: 1) symptoms of heart failure, defined as NYHA class II-IV, for at least 6 months prior to CRT, 2) LVEF $<35 \%$, and 3) QRS complex for a duration longer than 130 ms. If the duration of the QRS complex was less than $150 \mathrm{~ms}$, interventricular mechanical delay would have to be longer than $40 \mathrm{~ms}$ and transaortic pre-ejection delay should be longer than $140 \mathrm{~ms}$ [2]. All patients underwent coronary angiography; those patients with significant stenosis of coronary arteries or previous myocardial infarction were not included in this study. All patients signed informed consent prior to inclusion in the study.

\section{Baseline assessment}

Echocardiography. Echocardiographic examinations were performed using a VIVID 7 echo machine (General Electric, Horten, Norway). Left ventricular end-systolic and end-diastolic diameters and LVEF were determined according to the recommendations of the American Society of Echocardiography [7]. Interventricular mechanical delay was calculated as the difference between transpulmonary and transaortic pre-ejection intervals. Values above $40 \mathrm{~ms}$ were considered a sign of interventricular dyssynchrony.

Dobutamine stress echocardiography. The DSE was performed in all patients in incremental stages lasting $5 \mathrm{~min}$ each, with an initial dose of $5 \mathrm{mg} / \mathrm{kg} / \mathrm{min}$, which was increased to $10 \mathrm{mg} /$ $\mathrm{kg} / \mathrm{min}$, and finally to $20 \mathrm{mg} / \mathrm{kg} / \mathrm{min}$. The infusion was discontinued before the maximum dose when $85 \%$ of the maximum predicted heart rate for that age group was reached (as recommended by the American Society of Echocardiography), or symptomatic non-sustained or sustained ventricular tachycardia was observed. $\beta$-Blockers were stopped $48 \mathrm{~h}$ before the dobutamine testing in all patients when it was possible. Wall motion score index and ejection fraction were evaluated as indices of left ventricular contractility. The LVEF was determined by Simpson's biplane formula. Left ventricular wall motion score index (WMSI) was calculated by using a 16-segment model of the left ventricle [8]. The CR was defined as the difference between the values of these indices obtained at the peak dobutamine dose during the test and the baseline values $[9,10]$.

6-minute walk test (6MWT). The walking test was performed indoors, along a flat, enclosed, 25-m-long corridor with a hard surface, according to the American Thoracic Society guidelines [11]. The patients were instructed to walk at a pace from relatively slow to slightly tiring.

\section{Pacemaker implantation}

All leads were placed transvenously via the subclavian route. A coronary sinus venogram was obtained using a balloon catheter, followed by insertion of the LV pacing lead. A coronary sinus electrode was placed in a posterolateral or lateral tributary vein of the coronary sinus with an overthe-wire system. The atrioventricular interval was set prior to the discharge according to the previously defined criteria [12].

\section{Follow-up}

Patients were examined at intervals of 3, 6 and 12 months after CRT device implantation. Assessment of NYHA class, 6MWT, and echocardiographic measurement of LV diameters and ejection fraction were performed at each visit. Patients whose ejection fraction improved to $50 \%$ or more and whose left ventricular end-systolic diameter (LVESD) decreased below $40 \mathrm{~mm}$ after 12 months of CRT were considered as super-responders [5].

After completion of the 12-month follow-up, patients were followed regularly at 6-month intervals in our outpatient clinic, or they were contacted by phone. The time to hospitalization for worsening of heart failure or death was determined for all patients.

\section{Statistical analysis}

All data are presented as the mean \pm SD value. Comparisons of clinical and echocardiographic 
indices between the groups were performed by Student's t-test, when the data were normally distributed. Receiver operating characteristic (ROC) curves were used to determine the cutoff points for dobutamine test indices of $C R$ which discriminate between super-responders and non-super-responders, as defined in the methodology section. Area under the curve (AUC) was calculated for both dobutamine test indices. Kaplan-Meier curves were constructed for the time to death or to hospitalization for worsening of heart failure in super-responders and non-super-responders.

\section{Results}

\section{Study population}

In the study group, 46 (83\%) patients were male and 9 (17\%) were female. The mean age was $59.3 \pm 10.4$ years. Out of the total number, 11 patients were in NYHA class IV, 40 patients in NYHA class III, and 4 in NYHA class II. The mean duration of QRS complex was $173.7 \pm 17.9$ ms; 4 patients had a non-specific intraventricular conduction delay, and 51 patients had typical left bundle block QRS morphology. Five patients had QRS duration between $130 \mathrm{~ms}$ and $150 \mathrm{~ms}$, and the others had QRS complex duration longer than 150 ms. Interventricular mechanical delay was $65.9 \pm 30.8 \mathrm{~ms}$.
A CRT-ICD device was implanted in 5 patients and a CRT pacemaker in the rest of the patients. Other baseline data are presented in Table I.

\section{Dobutamine stress echocardiography}

$\beta$-Blockers were stopped in 54 patients $48 \mathrm{~h}$ prior to testing. During DSE the heart rate increased from $75.4 \pm 11.5$ beats/min at baseline to $107.6 \pm 18.1$ beats/min at the peak dose of dobutamine. Similarly, systolic blood pressure rose from $128 \pm 22 \mathrm{~mm} \mathrm{Hg}$ at baseline to $156 \pm 31 \mathrm{~mm} \mathrm{Hg}$ at the end of the test, whereas diastolic blood pressure rose from $82 \pm 15 \mathrm{~mm} \mathrm{Hg}$ to $94 \pm 15 \mathrm{~mm} \mathrm{Hg}$. During the test, two patients had single premature ventricular beats, and 1 patient had a short run of 3 premature ventricular beats. None of the patients experienced chest pain, hypotension or hypertension. WMSI changed from $2.6 \pm 0.3$ at baseline to $2.1 \pm 0.4$ at the peak dose of dobutamine $(p<0.001)$, whereas LVEF changed from $16.9 \pm 7.3 \%$ to $26.8 \pm 10.2 \%(p<0.001)$.

\section{Follow-up}

The mean duration of the follow-up in our study group was $28.5 \pm 3.0$ months. Four patients died during the follow-up, and 14 patients were hospitalized for worsening of heart failure symptoms. Seven patients fulfilled previously defined criteria for a super-response to CRT. Baseline clinical

Table I. Baseline clinical and echocardiographic characteristics

\begin{tabular}{|c|c|c|c|c|}
\hline Parameter & All patients & $\begin{array}{l}\text { Super-responders } \\
\qquad(n=7)\end{array}$ & $\begin{array}{c}\text { Non-super- } \\
\text { responders }(n=48)\end{array}$ & Value of $p^{*}$ \\
\hline Age & $59.3 \pm 10.4$ & $60.9 \pm 10.2$ & $59.1 \pm 10.5$ & 0.68 \\
\hline Female gender & $9(16.4 \%)$ & $2(28.6 \%)$ & $7(14.6 \%)$ & 0.32 \\
\hline NYHA class & $3.0 \pm 0.5$ & $2.6 \pm 0.2$ & $3.1 \pm 0.5$ & 0.04 \\
\hline LBBB & 51 (92.7\%) & $7(100 \%)$ & $44(91.6 \%)$ & 0.42 \\
\hline AF & $9(16.4 \%)$ & $0(0 \%)$ & $9(18.8 \%)$ & 0.21 \\
\hline QRS duration [ms] & $173.7 \pm 17.9$ & $192.3 \pm 20.1$ & $171.0 \pm 16.04$ & 0.03 \\
\hline $6 \mathrm{MWT}[\mathrm{m}]$ & $317.5 \pm 49.4$ & $369.4 \pm 22.0$ & $309.7 \pm 47.7$ & $<0.01$ \\
\hline LVEDD [mm] & $69.8 \pm 8.9$ & $62.7 \pm 2.4$ & $70.8 \pm 9.0$ & 0.02 \\
\hline LVESD [mm] & $53.7 \pm 11.6$ & $42.9 \pm 4.3$ & $55.2 \pm 11.5$ & $<0.01$ \\
\hline LVEF [\%] & $16.9 \pm 7.3$ & $19.4 \pm 5.4$ & $16.5 \pm 7.5$ & 0.33 \\
\hline RVEDD & $23.7 \pm 6.5$ & $25.1 \pm 0.9$ & $23.5 \pm 6.9$ & 0.54 \\
\hline$M R$ & $1.5 \pm 1.3$ & $0.6 \pm 0.8$ & $1.7 \pm 1.3$ & 0.03 \\
\hline$\Delta \mathrm{WMSI}$ & $0.57 \pm 0.39$ & $1.031 \pm 0.120$ & $0.49 \pm 0.371$ & $<0.01$ \\
\hline$\Delta \mathrm{EF}$ & $9.95 \pm 6.541$ & $17.857 \pm 2.1931$ & $8.792 \pm 6.152$ & $<0.01$ \\
\hline
\end{tabular}

$\angle B B B$ - left bundle branch block, AF - atrial fibrillation, 6MWT - 6-minute walking test, LVEDD - left ventricular end-diastolic dimension, LVESD - left ventricular end-systolic dimension, LVEF - left ventricular ejection fraction, RVEDD - right ventricular end-diastolic dimension, $M R$ - mitral regurgitation, $\triangle W M S I$ - wall motion score index change during the dobutamine test, $\triangle E F$ - ejection fraction change during the dobutamine test; " $p$ value corresponds to relation between super-responders and non-super-responders 
and echocardiographic data were compared between super- and non-super-responders (Table I). Super-responders had significantly better indices for CR, as well as longer QRS duration, lower NYHA class and better results in the 6-minute walk test. They also tended to have smaller diameters of the heart, and less pronounced mitral regurgitation.

During the entire follow-up period, patients with a super-response to CRT neither died nor were hospitalized for worsening of heart failure. However, there was no statistically significant difference between super- and non-super-responders in composite end-point of cardiac death and hospitalization for heart failure, as shown by the Kaplan-Maier curve (Figure 1).

The ROC curves were constructed for the dobutamine-induced $\triangle \mathrm{WMSI}$ and $\triangle \mathrm{EF}$ changes, and they identified that the best discriminator for super-response was dobutamine-induced change by $14 \%$ in $\triangle \mathrm{EF}$, and 0.7 for $\triangle \mathrm{WMSI}$ (Figure 2). Area under the curve was 0.906 for $\Delta \mathrm{EF}$, and 0.915 for $\Delta \mathrm{WMSI}$. For the cutoff value of 0.7 for $\Delta \mathrm{WMSI}$ the sensitivity is $100 \%$, specificity $67 \%$, positive predictive value $31 \%$ and negative predictive value $100 \%$; for the cutoff value of $14 \%$ for $\Delta E F$, the sensitivity is $100 \%$, specificity $75 \%$, negative predictive value $100 \%$ and positive predictive value $35 \%$.

The patients with $\Delta \mathrm{EF}>14 \%$ and $\Delta \mathrm{WMSI}>0.7$ during DSE had significantly better survival chances free of hospitalization for worsening of heart failure, as shown by the Kaplan-Maier curves (Figures 3 and 4).
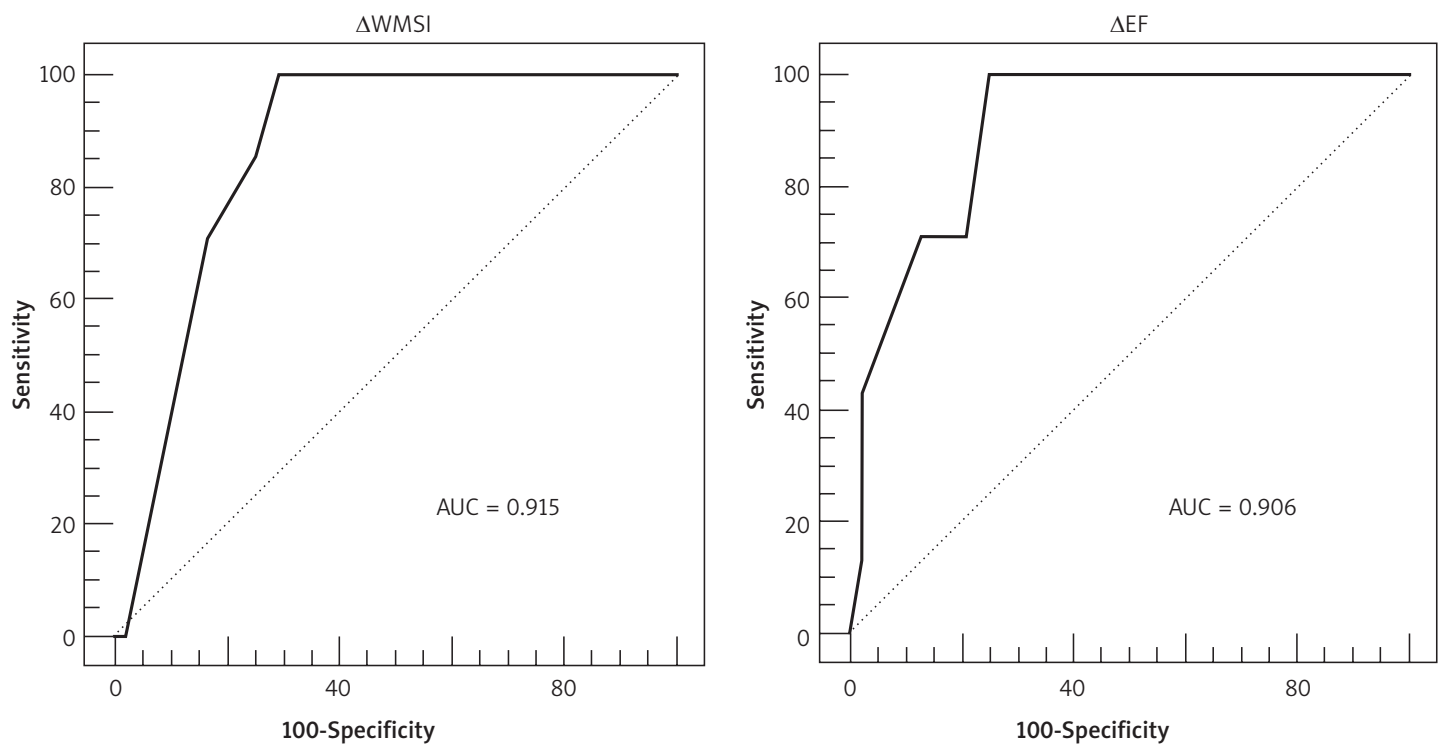

Figure 2. Receiver operating characteristic curve for wall motion score index change (A) and ejection fraction change during dobutamine test (B)

AUC - area under curve

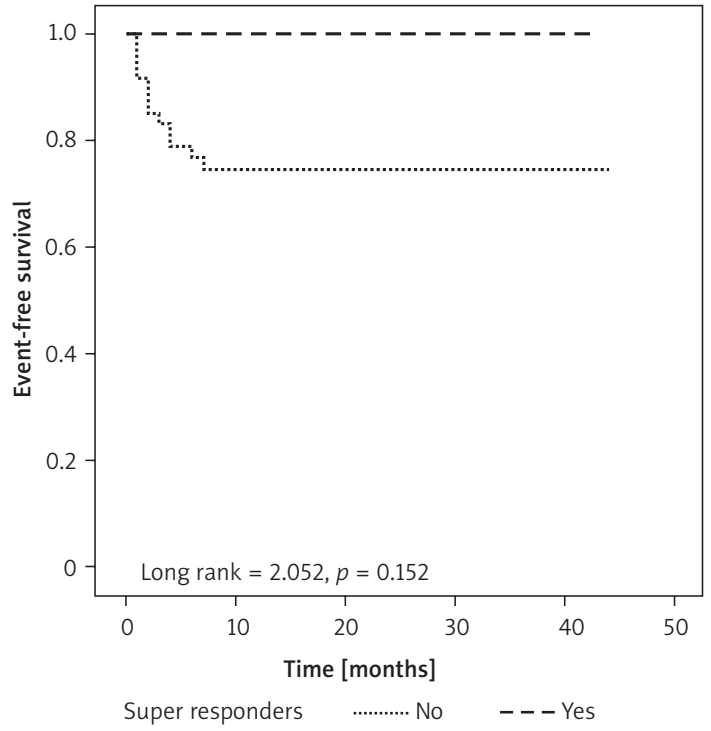

Figure 1. Kaplan-Maier curves showing the survival free of rehospitalization for worsening of heart failure, according to the super-response to the resynchronization therapy

\section{Discussion}

The main finding of our study is that CR detected during a dobutamine test predicts nearly complete reverse remodeling in patients with dilated cardiomyopathy treated with CRT pacemakers. In patients with dilated cardiomyopathy and left bundle branch block, in whom the wall motion score index decreases by more than 0.7 , and ejection fraction increases by at least $14 \%$, at the dobutamine dose of $20 \mu \mathrm{g} / \mathrm{kg} / \mathrm{min}$, cardiac 


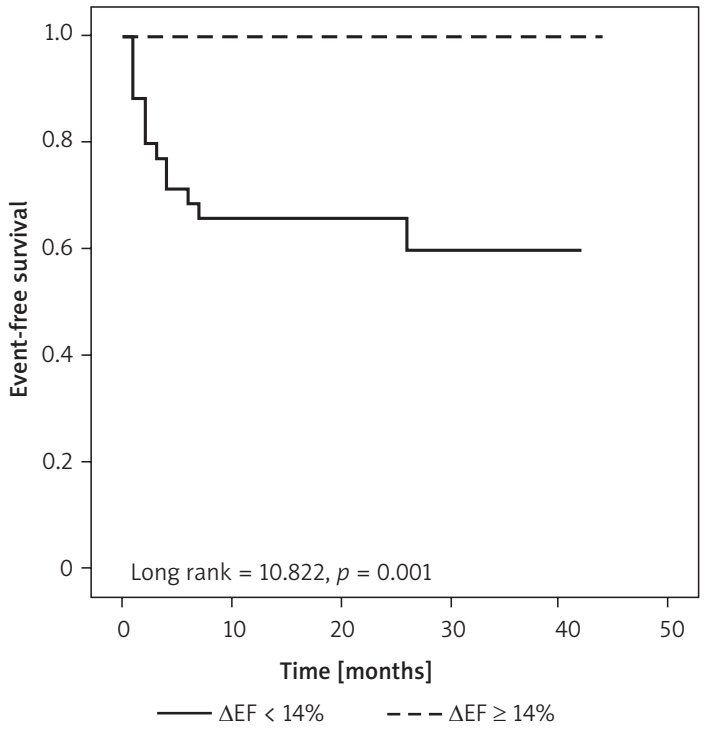

Figure 3. Kaplan-Maier curves showing the survival free of rehospitalization for worsening of heart failure, according to the dobutamine-induced change in left ventricular ejection fraction

resynchronization therapy may result in almost complete recovery of left ventricular systolic function. To the best of our knowledge, this is the first study to demonstrate the value of dobutamine stress echocardiography not only for predicting response to CRT, but also for predicting full recovery under this form of heart failure therapy.

Resynchronization therapy has become an established treatment option for selected patients with impaired systolic LV function and delayed and asynchronous LV contraction. The implantation of a CRT pacemaker reduces the symptoms of heart failure, improves the exercise capacity and prolongs the life of such patients. However, not every patient has the same benefit of cardiac resynchronization. Echocardiography may help optimize CRT, by enabling programming atrioventricular and interventricular delay with best hemodynamic response [13]. Furthermore, it plays an important role in detecting patients who are more likely to respond favorably to resynchronization therapy. Various echocardiographic indices of interventricular and intraventricular mechanical asynchrony have been tested for the prediction of response to CRT. The results of the PROSPECT study are somewhat disappointing, when indices of asynchrony, such as Doppler tissue imaging parameters, are used for this purpose [14]. Attempts have been made to develop a scoring system which combines different echocardiographic and electrocardiographic parameters, in order to better predict the outcome of resynchronization therapy [15]. On the other hand, the presence of myocardial contractile reserve assessed by the dobutamine test [16,17], exercise test [18], or mag-

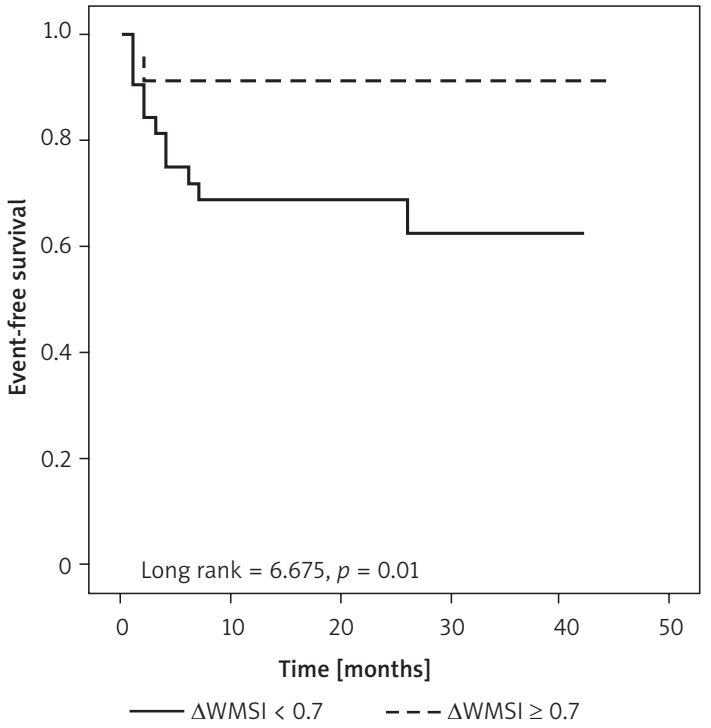

Figure 4. Kaplan-Maier curves showing the survival free of rehospitalization for worsening of heart failure, according to the dobutamine-induced change in wall motion score index

netic resonance imaging [19], appears to identify patients who are likely to show a functional and echocardiographic improvement. The result of our study extends these observations, and suggests that patients with a highly positive dobutamine test are likely to recover completely on the resynchronization therapy.

It is well known that the density and responsiveness of $\beta$-adrenergic-receptors decrease in patients who suffer from heart failure [20-22]. The improvement of contractile reserve indices during dobutamine infusion may be linked to the less advanced down-regulation of beta receptors on cardiomyocyte membranes [23]. On the other hand, it has been shown that some parameters of histological disruption such as myocyte diameter and the degree of interstitial fibrosis, usually found in the biopsy specimen of patients with cardiomyopathy, strongly correlate with the change of WMSI and LVEF during the high-dose dobutamine test [24]. We believe that patients with a highly positive dobutamine test for contractile reserve have "less damaged" hearts than those patients without contractile reserve and thus they may be more likely to completely recover on CRT.

In our study we used an intermediate dobutamine dose of up to $20 \mu \mathrm{g} / \mathrm{kg} / \mathrm{min}$, in order to avoid serious ventricular arrhythmias, which may occur in more than $10 \%$ of patients with heart failure when tested with the high dose $(40 \mu \mathrm{g} / \mathrm{kg} / \mathrm{min})$ [16]. On the other hand, it is possible that the use of low dose dobutamine $(2.5-5 \mu \mathrm{g} / \mathrm{kg} / \mathrm{min})$ would increase specificity, but certainly miss some potential super-responders and reduce sensitivity of this method. 
Previous studies and predictors

of "super-response" to cardiac resynchronization therapy

Super-responders or hyper-responders to cardiac resynchronization therapy are first mentioned in a case report of Bulawa, who described a case of a 72-year-old woman with non-ischemic cardiomyopathy, who experienced a complete recovery of LV function in the course of 1 year of CRT [25]. Blanc described a series of 29 patients with dilated cardiomyopathy, where the same phenomenon was observed in 5 patients [26] Several other smaller cohorts of patients were analyzed regarding the predictors of super-response to CRT. Castellant et al. [4] found that a complete recovery of LV function, along with a significant functional improvement, occurs more frequently in patients with dilated cardiomyopathy. In a series of 85 patients with dilated cardiomyopathy, 11 patients were termed "hyper-responders", and all belonged to the group with non-ischemic dilated cardiomyopathy. Due to the presence of an extensive myocardial scar after myocardial in farction, super-response to CRT defined as nearly complete recovery of $\mathrm{LV}$ systolic function probably could not be expected in patients with ischemic cardiomyopathy. The authors further hypothesized that left bundle branch block may even be a causative factor for the heart disease, at least in a subset of patients. A striking finding in our study is that super-responders were those patients with relatively preserved mechanical function (positive test for contractile reserve, smaller hearts and better baseline 6MWT), and worse electrical dyssynchrony, out of proportion to mechanical dysfunction. It is possible that in such patients the resynchronization of LV contraction removes dyssynchrony as the causative factor for the disease and allows complete recovery of the patient. In a retrospective analysis of 233 patients, Rickard [5] found that female gender, lower serum brain natriuretic peptide and typical LBBB pattern were significantly more frequent among patients with a super-response. Antonio [27] studied 87 patients with an established indication for CRT, and found that $12 \%$ of patients had a hyper-response to CRT, defined as twofold or greater increase in LVEF and $>15 \%$ decrease in LV end-systolic volume. According to their work, patients with a shorter duration of heart failure symptoms and less altered ventricular geometry seem to stand more chance of a nearly complete recovery under resynchronization therapy. Similarly, Reant et al. found that patients with lower left ventricular dimensions and greater global longitudinal strain, both factors reflecting better LV function, are more likely to have a super-response to CRT [6].
We found that 7 patients (12.7\%) fulfilled the criteria for a super-response to CRT, a percentage similar to that from other studies. A trend to a higher survival rate free of hospitalization for worsening of heart failure was noted in the super-responder group when compared to the other patients; the statistical significance of this difference found in other studies [5] was not reached in our cohort, probably due to its small size. We did not find that female gender or a typical LBBB pattern correlates with super-responsiveness to CRT. Only 9 women were included in our study cohort, which might explain our results. Similarly, the vast majority of our patients have a typical LBBB pattern, which precludes any relevant statistical analysis. We believe that parameters such as less altered ventricular geometry, lower LV dimensions, greater global longitudinal strain and preserved contractile reserve are in accordance with each other.

\section{Clinical implications}

We have shown that patients with dilated cardiomyopathy, who have wide QRS and a highly positive dobutamine test, are more likely to experience complete restoration of LV function. In our opinion, patients with dilated cardiomyopathy who are candidates for CRT should undergo the estimation of contractile reserve and those who have a highly positive DSE should receive this type of therapy without unnecessary delay, because a prolonged illness and more pronounced LV remodeling might affect the positive effects of CRT, and make a super-response unlikely to occur. This observation is in accordance with the results of the MADIT-CRT [28] and REVERSE [29] trials, which implies that the implantation of a CRT pacemaker in the earlier stages of the disease in heart failure patients with asynchronous contraction is beneficial, favoring reverse remodeling of the left ventricle and reducing morbidity.

\section{Limitations of the study}

The main limitation of the present single centre study is the small size of the group. We included a relatively small proportion of female patients. The predictive value of the dobutamine test has to be confirmed in a larger series of patients, with more female participants. In addition, LV ejection fraction as well as its change and the change in WMSI during the dobutamine test were determined by the same physicians who followed up the patients, i.e. they were not blinded to the effect of CRT. On the other hand, LVEF is not an ideal echocardiographic parameter, and it is subject to pre-load and after-load influences, which limits its use as a reliable parameter of reverse remodeling. 
The patients from our study are from a single tertiary referral centre and might not represent a typical CRT candidate. We chose cutoff values for the indices of contractile reserve by inspection of the ROC curves only after the study had been completed, and afterwards we tested them using the Kaplan-Meier analysis. A prospective study with cutoff values defined prior to patient inclusion would yield much stronger results. Multicenter studies are warranted to prove the value of a dobutamine test in predicting a super-response to CRT.

In conclusion, contractile reserve induced by dobutamine stress echocardiography can identify patients who are likely to have near normalization of LV function following CRT.

\section{References}

1. Bristow MR, Saxon LA, Boehmer J, et al.; for the Comparison of Medical Therapy, Pacing, and Defibrillation in Heart Failure (COMPANION) Investigators. Cardiac-resynchronization therapy with or without an implantable defibirllatior in advanced chronic heart failure. $\mathrm{N}$ Eng J Med 2004; 350: 140-50.

2. Cleland JGF, Daubert JC, Erdmann E, et al.; the Cardiac Resynchronization - Heart Failure (CARE-HF) Study Investigators. The effect of cardiac resynchronization on Morbidity and Mortality in Heart Failure. N Engl J Med 2005; 352: 1539-49.

3. Da Costa A, Thévenin J, Roche F, et al. Prospective validation of stress echocardiography as an identifier of cardiac resynchronization therapy responders. Heart Rhythm 2006; 3: 406-13.

4. Castellant P, Fatemi M, Bertault-Valls V, Etienne Y, Blanc JJ. Cardiac resynchronization therapy: "nonresponders"and "hyperresponders". Heart Rhythm 2008; 5: 193-7.

5. Rickard J, Kumbhani DJ, Popovic Z, et al. Characterization of super-response to cardiac resynchronization therapy. Heart Rhythm 2010; 7: 885-9.

6. Reant P, Zaroui A, Donal E, et al. Identification and characterization of super-responders after cardiac resynchronization therapy. Am J Cardiol 2010; 105: 1327-35.

7. Lang RM, Bierig M, Devereux RB, et al. Recommendations for Chamber Quantification: a Report from the American Society of Echocardiography's Guidelines and Standards Committee and the Chamber Quantification Writing Group, Developed in Conjunction with the European Association of Echocardiography, a Branch of the European Society of Cardiology. I Am Soc Echocardiogr 2005; 18: 1440-63.

8. Pelikka PA, Nagueh SF, Elhendy AA, et al. American society of echocardiography. Recommendations for performance, interpretation and application of stress echocardiography. J Am Soc Echocardiogr 2007; 20: 1021-41.

9. Schiller NB, Shah PM, Crawford M, et al. Recommendations for quantitation of the left ventricle by two-dimensional echocardiography. American Society of Echocardiography Committee on Standards, Subcommittee on Quantitation of Two-Dimensional Echocardiograms. J Am Soc Echocardiogr 1989; 2: 358-67.

10. Otasevic P, Popovic ZB, Vasiljevic JD, et al. Head-to-head comparison of indices of left ventricular contractile reserve assessed by high-dose dobutamine stress echocardiography in idiopathic dilated cardiomyopathy: fiveyear follow up. Heart 2006; 92: 1253-8.
11. American Thoracic Society. ATS statement: guidelines for the six-minute walk test. Am J Respir Crit Care Med 2002; 166: 111-7.

12. Linde C, Gadler F, Edner M, Nordlander R, Rosenquist M, Ryden L. Result of atrioventricular synchronous pacing with optimized delay in patients with severe congestive heart failure. Am J Cardiol 1995; 75: 919-23.

13. Dabrowska-Kugacka A, Lewicka E, Faran A, et al. Righr atrial appendage pacing in cardiac resynchronization therapy - haemodynamic consequences of interatrial conduction delay. Arch Med Sci 2011; 7: 728-31.

14. Chung ES, Leon AR, Tavazzi L, et al. Results of the predictors of response to CRT (PROSPECT) trial. Circulation 2008; 117: 2606-18.

15. Shen X, Nair CK, Aronow WS, et al. A new baseline scoring system may help to predict response to cardiac resynchronization therapy. Arch Med Sci 2011; 7: 627-33.

16. Ciampi Q, Pratali L, Citro R, Piacenti M, Villari B, Picano E. Identification of responders to cardiac resynchronization therapy by contractile reserve during stress echocardiography. Eur J Heart Fail 2009; 11: 489-96.

17. Parsai C, Baltabaeva A, Anderson L, Chaparro M, Bijnens B, Sutherland GR. Low-dose dobutamine stress echo to quantify the degree of remodelling after cardiac resynchronization therapy. Eur Heart J 2009; 30: 950-8.

18. Lancellotti P, Senechal M, Moonen M, et al. Myocardial contractile reserve during exercise predicts left ventricular reverse remodelling after cardiac resynchronization therapy. Eur J Echocardiogr 2009; 10: 663-8.

19. Ypenburg C, Roes SD, Bleeker GB, et al. Effect of total scar burden on contrast-enhanced magnetic resonance imaging on response to cardiac resynchronization therapy. Am J Cardiol 2007; 99: 657-60.

20. Bristow MR, Ginsburg R, Minobe W, et al. Decreased cateholamine sensitivity and beta-adrenergic-receptor density in failing human hearts. N Engl J Med 1982; 307: 205-11.

21. Ginsburg R, Bristow MR, Billingham ME, Stinson EB, Schroeder JS, Harrison DC. Study of the normal and failing isolated human heart: decreased response of failing heart to isoprotenorol. Am Heart J 1983; 106: 535-42.

22. Bristow MR. The adrenergic nervous system in heart failure. N Engl J Med 1984; 311: 850-9.

23. Leier CV, Heban PT, Huss P, Bush CA, Lewis RP. Comparative systemic and regional hemodynamic effects of dopamine and dobutamine in patients with cardiomyopathic heart failure. Circulation 1978; 58: 466-72.

24. Otasević P, Popović ZB, Vasiljević JD, et al. Relation of myocardial histomorphometric features and left ventricular contractile reserve assessed by high-dose dobutamine stress echocardiography in patients with idiopathic dilated cardiomyopathy. Eur J Heart Fail 2005; 7: 49-56.

25. Bulava A, Lukl J, Skvarilová M, Marek D. Dramatically improved left ventricular function after biventricular pacemaker implantation-a case report. Eur J Heart Fail 2005; 7: 231-3.

26. Blanc JJ, Fatemi M, Bertaul-Valls V, Baraket F, Etienne Y. Evaluation of left bundle branch block as a reversible cause of non-ischaemic dilated cardiomyopathy with severe heart failure. A new concept of left ventricular dyssynchrony induced cardiomyopathy. Europace 2005; 7: 604-10.

27. Antonio N, Teixeira R, Coelho, et al. Identification of "super-responders" to cardiac resynchronization therapy: 
the importance of symptom duration and left ventricular geometry. Europace 2009; 11: 343-9.

28. Moss AJ, Jackson Hall W, Cannom DS, et al.; the MADIT-CRT Trial Investigators. Cardiac-resynchronization therapy for the prevention of heart-failure events. N Engl J Med 2009; 361: 1329-38.

29. Sutton MSJ, Ghio S, Plappert T, et al.; the REsynchronization reVErses Remodeling in Systolic left vEntricular dysfunction (REVERSE) Study Group. Cardiac Resynchronization Induces Major Structural and Functional Reverse Remodeling in Patients With New York Heart Association Class 1/II Heart Failure. Circulation 2009; 120: $1858-65$ 